\title{
Effect of lattice-gas atoms on the adsorption behaviour of thioether molecules
}

\author{
Yi Pan, ${ }^{a}$ Bing Yang, ${ }^{a}$ Catherine Hulot, ${ }^{b}$ Siegfried Blechert, ${ }^{b}$ Niklas Nilius* ${ }^{a}$ and \\ Hans-Joachim Freund ${ }^{a}$
}

Received 4th April 2012, Accepted 7th June 2012

DOI: $10.1039 / \mathrm{c} 2 \mathrm{cp} 41081 \mathrm{~h}$

Using STM topographic imaging and spectroscopy, we have investigated the adsorption of two thioether molecules, 1,2-bis(phenylthio)benzene and (bis(3-phenylthio)-phenyl)sulfane, on noble and transition metal surfaces. The two substrates show nearly antipodal behaviour. Whereas complexes with one or two protruding centres are observed on $\mathrm{Au}(111)$, only flat and uniform ad-structures are found on $\mathrm{NiAl}(110)$. The difference is ascribed to the possibility of the thioethers to form metal-organic complexes by coordinating lattice-gas atoms on the Au(111), while only the pristine molecules adsorb on the alloy surface. The metal coordination in the first case is driven by the formation of strong $\mathrm{Au}-\mathrm{S}$ bonds and enables the formation of characteristic monomer, dimer and chain-like structures of the thioethers, using the $\mathrm{Au}$ atoms as linkers. A similar mechanism is not available on the $\mathrm{NiAl}$, because no lattice gas develops at this surface at room temperature. Our work demonstrates how surface properties, i.e. the availability of mobile ad-species, determine the interaction of organic molecules with metallic substrates.

\section{Introduction}

Organic molecules that are composed of aryl groups connected via sulphur atoms are known for their strong interaction with metal surfaces. ${ }^{1-5}$ The binding mechanism relies on two effects, which are the formation of covalent sulphur-metal bonds and the overlap of the $\pi$-system of the aryls and the de-localized electronic states on the metal surface. However, only recently it has been realized that the flat metal surface does not provide the energetically preferred binding template, but individual metal adatoms are responsible for coordinating the S-centres of the molecules. ${ }^{4,6,7}$ This insight, gained by combined experimental and theoretical efforts, changed the overall perception of the interplay between metal surfaces and a wide range of organic molecules, including alkane-thiolates, thio-phenol and thioether compounds. ${ }^{1,8-10}$ Moreover, the ability of organic ligands to coordinate metal adatoms has initiated a completely new research field that focuses on the development of well-ordered metalorganic frameworks. The associated self-organization phenomena open interesting routes to pattern and functionalize metal surfaces and to generate assemblies for molecular electronic devices. ${ }^{11-14}$

Another potential application of sulphur-containing aryl compounds has been widely disregarded so far. By trapping metal adatoms, such molecules might be exploited to control

${ }^{a}$ Fritz-Haber-Institut der Max-Planck-Gesellschaft, Faradayweg 4-6, D-14195 Berlin, Germany

${ }^{b}$ Technische Universität Berlin, Institut für Chemie, Straße des 17. Juni 135, D-10623 Berlin, Germany.

E-mail: nilius@fhi-berlin.mpg.de diffusion processes on solid surfaces, which take place for instance between metal ad-particles on oxide surfaces. This undesirable material flow gives rise to sintering and ripening processes ${ }^{15}$ and is in part responsible for the deactivation of heterogeneous catalysts used in the chemical industry. ${ }^{16}$ The fundamental reason for this reactivity decline is the gradual transformation of small and highly active aggregates into bulk-like and inert deposits at the temperature and pressure conditions prevailing in a chemical reaction. Inhibiting or even reversing ripening processes on a catalyst surface is thus an issue of technological importance and has been in the focus of catalysis research for many decades. ${ }^{16}$ Sulphur containing organic molecules could become relevant in this respect, as they are able to trap and coordinate mobile adatoms and hence alter diffusion processes on oxide supports. ${ }^{17}$ To fully exploit the complexation potential of the thioethers, atomtrapping processes need to be explored as a function of temperature, ligand concentration and chemical environment, preferentially on the atomic scale. The derived information might help in designing suitable ligand molecules and revealing thermodynamic parameters for their application.

Using scanning tunnelling microscopy (STM), we have explored the ability of two thioether species to coordinate single metal adatoms on $\mathrm{Au}(111)$ and $\mathrm{NiAl}(110)$ surfaces. The adatoms are supplied by the lattice-gas that is intrinsically present on many metal surfaces and results from the continuous attachment/detachment of atoms from surface step edges. ${ }^{18}$ The density of the lattice-gas mainly depends on the thermodynamic stability of the exposed steps and their straightness, i.e. the 
number of low-coordinated kink and corner sites, and is expected to vary for different substrates. Moreover, the density of mobile ad-species changes with the surface temperature, as atom detachment from a step edge is an activated process. ${ }^{19}$ In this work, we have analysed the nature of adsorption complexes that develop upon trapping of single metal adatoms by the thioether molecules. By comparing the number of incorporated metal atoms, we discuss the suitability of different thioethers for metal complexation and the role of the metal substrate in such processes. We note that the metal lattice-gas might be considered as a model system to mimic atom-exchange processes between metal particles on oxide surfaces. Details of the complexation processes obtained here might therefore provide input parameters for a molecular-driven redispersion scheme, as envisioned in heterogeneous catalysis.

\section{Ligand preparation}

Two thioether species have been synthesized in our study, namely 1,2-bis(phenylthio)benzene and (bis(3-phenylthio)-phenyl)sulfane, referred to as BPB and BPPS in the following. ${ }^{20,21}$ Both compounds consist of chains of phenyl rings connected via $\mathrm{S}$-atoms (Fig. 1). In BPB, one central benzene coordinates two outer phenyls via two sulphurs in ortho-positions, while the BPPS comprises four phenyls that are linked by three S-atoms in meta-positions. Thanks to possible rotations about the $\mathrm{S}-\mathrm{C}$ axes, the BPPS has a larger structural flexibility, which makes the S-centres better accessible for adsorbates. We thus expect a higher complexation potential for BPPS than BPB molecules.

$\mathrm{BPB}$ has been synthesized according to a procedure developed by Bates et al., ${ }^{22}$ using 1,2-diiodobenzene as starting substance. The reaction yield was as high as $99 \%$ and the product could be easily purified via multiple chromatographic columns followed by sublimation cycles. The synthesis of the BPPS was more challenging and only a recipe by Hiroshi et al., ${ }^{23}$ being optimized by modifying solvent, reaction time and temperature, gave acceptable yields of $\sim 50 \%$. The synthesis was carried out by reacting $N$-methyl-2-pyrrolidone at $500 \mathrm{~K}$ under microwave irradiation. Also purification of the BPPS turned out to be difficult and common schemes, such as conventional and radial chromatography, normal and reverse HPLC and sublimation, failed because of similar retention times and molecular weights of the impurities. A clean substance was finally obtained by degassing the molecules under ultra-high vacuum (UHV) conditions at temperatures just below the sublimation point $(430 \mathrm{~K})$. After cleaning, the molecules were vapour deposited from two separate Knudsen cells onto $\mathrm{Au}(111)$ and $\mathrm{NiAl}(110)$ at room temperature. The metal surfaces had been cleaned
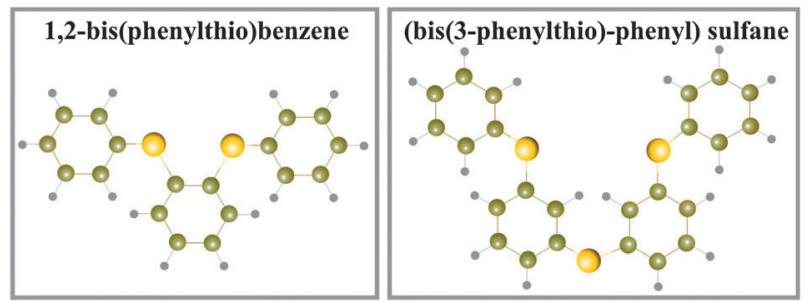

Fig. 1 Structure model of 1,2-bis(phenylthio)benzene (BPB) and (bis(3-phenylthio)-phenyl)sulfane (BPPS) used in this study. before by sputter/anneal cycles, until sharp spot patterns and atomically-flat surfaces were obtained in low-energy-electron diffraction and STM, respectively. After deposition, the samples were immediately transferred into a helium-cooled STM in order to suppress adsorption from the residual gas. The resulting ad-features were characterized by constant current and differential conductance imaging at $10-50 \mathrm{pA}$ electron current.

\section{Results and discussion}

\subsection{Molecular adsorption on the Au(111) surface}

We will start our discussion with the gold substrate that provides an excess of mobile adatoms thanks to a dense, room-temperature lattice gas. ${ }^{18}$ The STM images in Fig. 2 show the $\mathrm{Au}(111)$ surface after dosing small amounts of the ligand molecules. Evidently, the ad-features exclusively appear in the fcc regions of the gold herringbone reconstruction, as well as on the upside of surface step edges. ${ }^{7}$ The most characteristic BPB and BPPS complexes have been selected for Fig. 3. In the upper three panels, straight and bent entities are shown that have an average length of 10 and $15 \AA$ for BPB and BPPS, respectively (Fig. 3a). The features are $\sim 1.5 \AA$ high, a value that is almost independent of the applied sample bias. Those entities are the smallest features found on the surface which is why we assign them to isolated molecules, an assumption that will be substantiated later. The complexes in the second row differ from those in the top panels by the presence of a $2.5 \AA$-high protrusion in the centre (Fig. 3b). While only one single-maximum feature is detected for BPB, two configurations are found for BPPS, namely an oval one with $16 \AA$ diameter and an elongated one with $23 \AA$ length and $10 \AA$ width. The latter locates preferentially at the elbows of the herringbone reconstruction. Because of the single maximum in the centre, we refer to the ad-structures in Fig. $3 \mathrm{~b}$ as monomers. The last types of complexes display two maxima and are therefore denoted as dimers (Fig. 3c). The two dimer protrusions have the same height as the single maximum of the monomer $(2.5 \AA)$ and are approximately $7 \AA$ apart. As for the monomer, only a single BPB dimer is found that has an elongated shape and a total length of $20 \AA$. In contrast, the BPPS forms two conformers, an elongated one being similar to the BPB dimer and an oval one with $20 \AA$ length and $16 \AA$ width. In general, the dimers are by far more
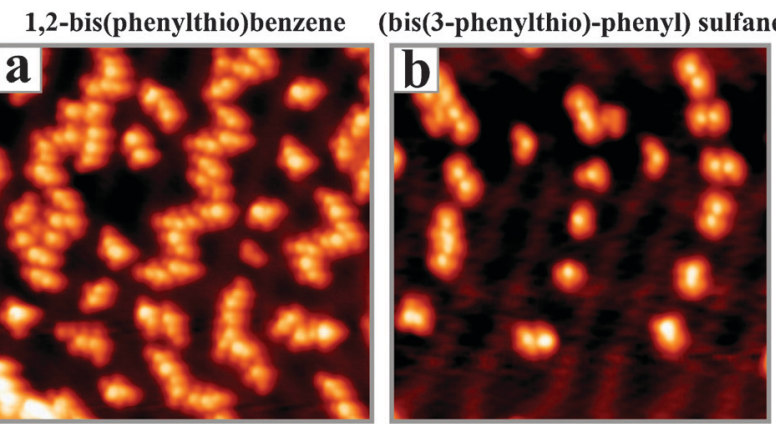

Fig. 2 STM overview images of (a) BPB and (b) BPPS taken on $\mathrm{Au}(111)$ at low molecular exposure $\left(22 \times 22 \mathrm{~nm}^{2}, U_{\mathrm{s}}=-0.5 \mathrm{~V}\right)$. The ligands bind preferentially to the fcc-region of the gold herringbone reconstruction. 


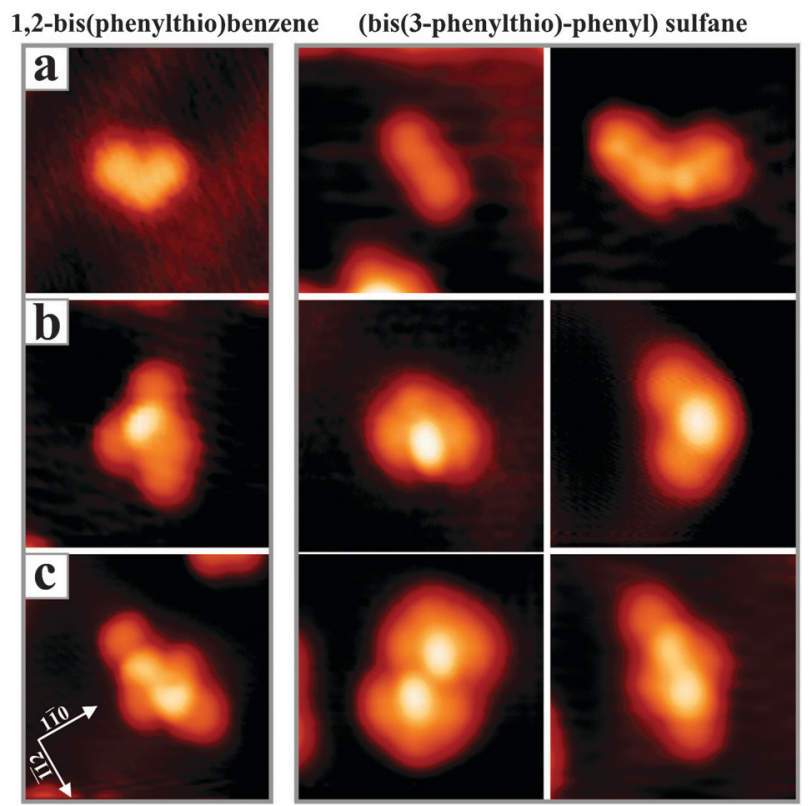

Fig. 3 STM images of different complexes observed on $\mathrm{Au}(111)$ at low molecular exposure. Left and right columns: BPB and BPPS molecules $\left(4 \times 4 \mathrm{~nm}^{2}, U_{\mathrm{s}}=0.5 \mathrm{~V}\right)$. The different rows display (a) pristine molecules, (b) monomer structures composed of a single ligand and (c) dimer structures comprising two ligands.

abundant than monomers and bare molecules that occur only in negligible quantities on the $\mathrm{Au}(111)$ surface (Fig. 2). We note that all molecular configurations described above can be found in three rotational orientations, as expected from the six-fold symmetry of the $\mathrm{Au}(111)$.

At higher exposure, both BPB and BPPS molecules agglomerate into chains that may reach several $\mathrm{nm}$ in length (Fig. 4). The chains follow the characteristic zig-zag pattern of the herringbone reconstruction, reflecting the preferred adsorption of both thioether molecules in the $\mathrm{Au}(111)$ fcc-region. For BPB, single

1,2-bis(phenylthio)benzene

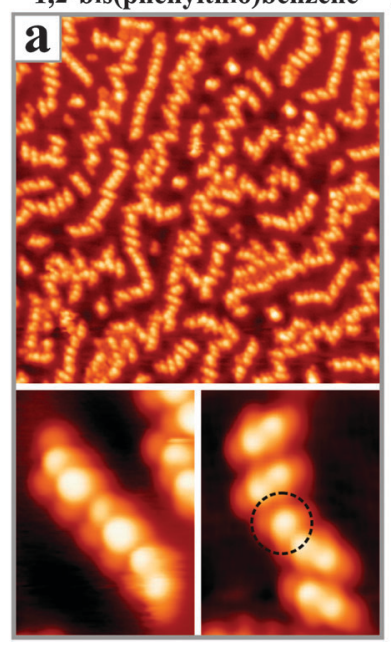

(bis(3-phenylthio)-phenyl) sulfane

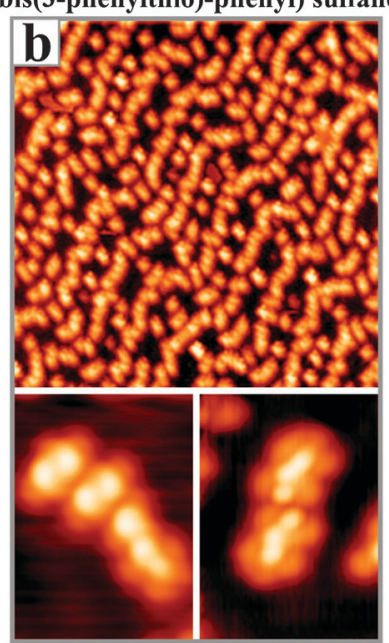

Fig. 4 STM images of the $\mathrm{Au}(111)$ surface taken at high exposure of (a) BPB and (b) BPPS molecules $\left(20 \times 20 \mathrm{~nm}^{2}, U_{\mathrm{s}}=-0.5 \mathrm{~V}\right)$. The insets show better resolved images of selected chain sections $\left(4.5 \times 6.5 \mathrm{~nm}^{2}\right)$. and double stranded chains are revealed that have either one or two protrusions perpendicular to the chain axis. Better resolved images enable the identification of individual building blocks of the BPB chains (Fig. 4a). Apparently, each chain is composed of BPB dimers, which are arranged either parallel to the chain axis (single stranded chain) or in a staggered configuration (double stranded chain). Occasionally, also BPB monomers are incorporated into the regular dimer pattern (Fig. 4a, circle). The area between adjacent chains is occupied only by individual monomer and dimer species, reflecting the low binding potential of the hcp-regions of the herringbone reconstruction.

The BPPS forms similar chains as the BPB, what the internal structure and the zig-zag course concerns (Fig. 4b). The chain sections are composed of dimer complexes as well, being arranged in a linear or staggered configuration with respect to the chain axis. Consequently, single and double stranded chains are observed again on the $\mathrm{Au}(111)$. Interestingly, only the elongated BPPS dimer is able to agglomerate into a molecular chain, while most of the oval species remain isolated. However, the latter is often found at the end of the BPPS chains, emphasizing their inability to coordinate other molecular units.

We note that disordered molecular islands develop on the $\mathrm{Au}(111)$ surface at even higher exposure. Those islands locate preferentially in between the chains and have a reduced topographic height with respect to the dimer structures. In fact, their height is compatible with the one of pristine molecules in Fig. 3a, indicating an island composition out of bare BPB and BPPS entities.

\subsection{Adsorption on the $\mathrm{NiAl}(110)$ surface}

$\mathrm{NiAl}$ was chosen as second substrate, because it shows nearly antipodal behaviour to gold. Its (110) surface has a bi-elemental composition consisting of alternating $\mathrm{Ni}$ and $\mathrm{Al}$ rows running along the [001] direction. ${ }^{24}$ Its rectangular unit-cell has four $\mathrm{Ni}$ atoms at the corners and one $\mathrm{Al}$ atom in the centre. The most prominent difference to gold is however the absence of a dense lattice-gas, which results from the strong interactions between the surface $\mathrm{Al}$ and $\mathrm{Ni}$ atoms. The modified surface properties of $\mathrm{NiAl}(110)$ are reflected in a deviating adsorption behaviour of the BPB molecules (Fig. 5a). Already at low exposure, a large variety of adsorption complexes is revealed in the STM images. The most common one is a $10 \AA$ long feature comprising one central and two outer maxima of 1.4 and $1.2 \AA$ height, respectively (Fig. 5c, upper left). With tips being functionalized with an organic molecule, the three-lobe structure transforms into a double protrusion with $4 \AA$ distance between the maxima (lower left). The long axis of the molecule preferentially aligns with the $\mathrm{NiAl}[1-10]$ direction while [001]-oriented molecules are rare. Individual species always coexist with small assemblies containing a few molecular building blocks. With increasing coverage, BPB agglomerates into larger 2D islands (Fig. 5c, right panels). However, their internal order is low and no superstructure was identified upon room temperature deposition.

The BPPS adsorption behaviour resembles that of the $\mathrm{BPB}$ in various aspects. The most common feature is a [1-10]-oriented rod of $13 \AA$ length that exhibits two outer maxima of $1.3 \AA$ apparent height and a central one that is 


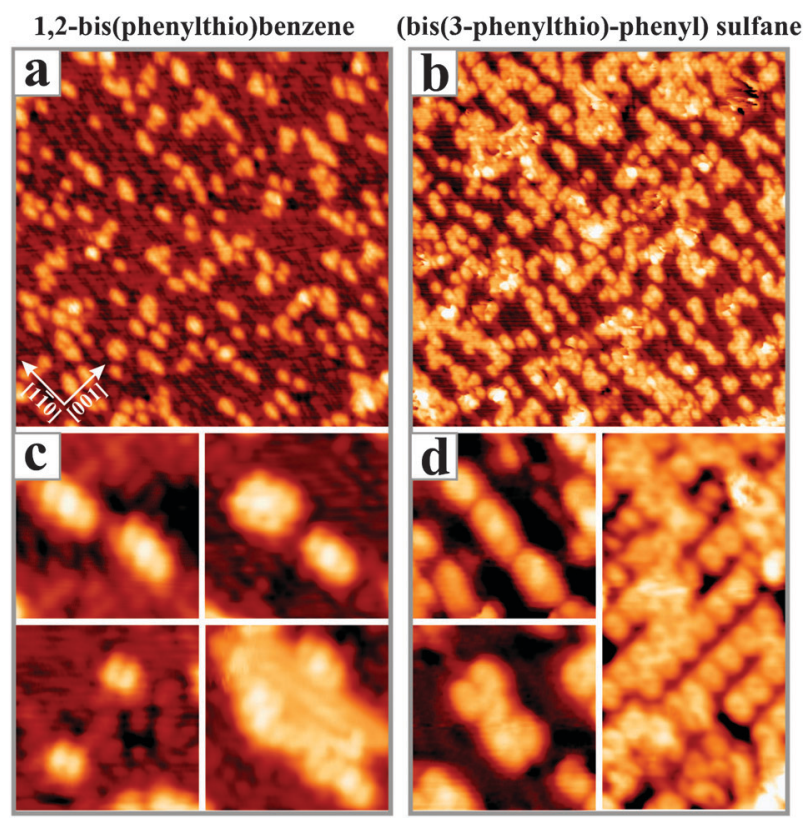

Fig. 5 STM images of the $\mathrm{NiAl}(110)$ surface taken after depositing $(\mathrm{a}, \mathrm{c})$ BPB and $(\mathrm{b}, \mathrm{d})$ BPPS molecules $\left(25 \times 25 \mathrm{~nm}^{2}, U_{\mathrm{s}}=-1.0 \mathrm{~V}\right)$. The insets show close-up images of individual molecular entities $\left(4 \times 4 \mathrm{~nm}^{2}\right)$ as well as the BPPS superstructure that forms at high exposure on the NiAl surface $\left(4 \times 8 \mathrm{~nm}^{2}\right)$.

taller by roughly $0.1 \AA$ (Fig. $5 \mathrm{~b}$ and d). Single adsorbates are randomly distributed on the $\mathrm{NiAl}(110)$ surface and form only small assemblies of two or three entities at low coverage. In contrast to the BPB, a molecular superstructure develops at higher exposure that comprises [001]-directed chains of [1-10]-oriented molecules (Fig. 5d, right panel). The superstructure is commensurate with the NiAl lattice, as adjacent molecules are separated by 5.8 and $8.2 \AA$ along the [001] and [1-10] direction of $\mathrm{NiAl}(110)$, corresponding to two unit cells in both cases. The superstructure fills large portions of the metal surface, before a second molecular layer starts growing on the top of the first one.

\subsection{Structure models for the different adsorbate complexes}

Characteristic adsorbate complexes have been observed on both the $\mathrm{Au}(111)$ and $\mathrm{NiAl}(110)$ surface, upon exposure to BPB and BPPS molecules at room temperature. In this chapter, we will rationalize the different STM topographs with the help of tentative binding models. The most obvious difference between the two supports is the apparent height of the molecules, which amounts to $2.5 \AA$ on $\mathrm{Au}(111)$ but hardly reaches $1.5 \AA$ on $\mathrm{NiAl}(110)$. Various reasons might be responsible for this difference. The binding geometry of the molecules and the level of hybridization with the metal electronic states could vary in both cases. We discard these options, because both substrates are metallic and should offer a similar density of free-electronlike states. Moreover, not even an upright standing phenyl would produce the $2.5 \AA$-elevation observed on gold, as weakly coupled molecular units are usually unsuitable for electron transport in the STM. ${ }^{17,25}$ We therefore favor another scenario, in which the thioether species are able to coordinate single adatoms on the $\mathrm{Au}(111)$ but not on the $\mathrm{NiAl}(110)$, a phenomenon that is explained by the different availability of a lattice gas on the two surfaces. ${ }^{26}$ We will substantiate this hypothesis in the following.

As demonstrated in earlier studies, ${ }^{2,4,8}$ sulphur-containing organic molecules exhibit a large affinity to coordinate $\mathrm{Au}$ atoms by forming covalent bonds between the $\mathrm{Au} 6 \mathrm{~s}^{1}$ and the S lone-pair electron. Alkanethiol chains, for example, were found to interact with $\mathrm{Au}(111)$ via an Au atom attached to the sulphur head of the chain. ${ }^{10}$ Also, thiophenyl and thioether molecules $^{4,17}$ are able to coordinate mobile ad-species and form metal-organic complexes. The effect of metal complexation is not only restricted to the $\mathrm{Au}-\mathrm{S}$ combination, but has also been found for other molecular end-groups, e.g. $\mathrm{CN}$, in conjunction with other metal atoms $(\mathrm{Co}, \mathrm{Fe}){ }^{27}$ Using this interaction scheme, the STM images obtained on the BPB and BPPS-covered gold surface can be interpreted. We will demonstrate all relevant steps towards a likely binding model for the oval BPPS monomer, and only sketch the final configurations for the other complexes.

The incorporation of $\mathrm{Au}$ atoms gives rise to the protruding centres in topographic images of BPPS monomer species (Fig. 6). Already a bare Au monomer and dimer are imaged with 1.5 and $2.0 \AA$ apparent height, respectively, as deduced from single-atom deposition experiments on the $\mathrm{Au}(111)$ surface. As shown in a recent DFT study, both values become larger upon $\mathrm{Au}-\mathrm{S}$ bond formation, because the S-containing molecular unit partly climbs the Au adatom in order to maximize the bond strength. ${ }^{28}$ Elucidating the exact Au position in a BPPS monomer complex is difficult from topographic images that integrate over all states between the preset bias voltage and the Fermi level. It can be achieved however in state-selected conductance maps, as shown in Fig. 6b-d. At $2.5 \mathrm{~V}$ sample bias, the single protrusion in the topography splits into two maxima that are $\sim 6 \AA$ apart in the conductance map (Fig. 6d). We assign this protruding pair to an $\mathrm{Au}$ dimer, which shows up in empty-state $\mathrm{d} I / \mathrm{d} V$ maps as mainly the antibonding Au 6s-type dimer-orbital governs the signal. ${ }^{29} \mathrm{At}$ negative polarity, only a single protrusion is revealed in the STM images, which reflects the bonding orbital that has highest density probability in between the Au-pair (Fig. 6a). In the corresponding $\mathrm{d} I / \mathrm{d} V$ maps, a minimum is detected because the large apparent height of the dimer at $-0.5 \mathrm{~V}$
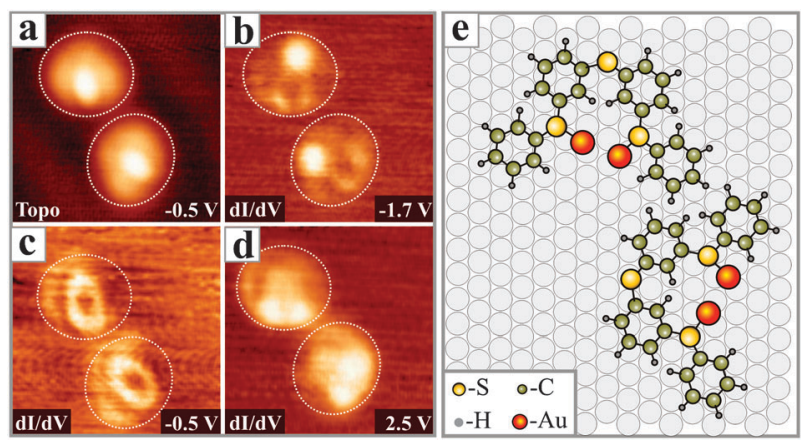

Fig. 6 Topographic (a) and conductance (b-d) images of an oval BPPS monomer taken at the given sample voltages $\left(5 \times 5 \mathrm{~nm}^{2}\right)$. The bright maxima in (b) and (d) are assigned to the uncoordinated S- and the Au-atoms in the complex, respectively. (e) Binding model of two BPPS monomers on $\mathrm{Au}(111)$ as deduced from the experimental data. 
reduces the overlap between tip and sample wave-functions and hence the $\mathrm{d} I / \mathrm{d} V$ signal (Fig. 6c). Our results indicate that BPPS is able to coordinate two Au atoms by forming bonds to its first and third S atom that are $5.4 \AA$ apart. Superimposing the $\mathrm{Au}(111)$ lattice on the STM images even allows us to determine the binding sites of the atom pair. Orientation and spacing of the two maxima are compatible with two Au atoms bound to second-neighbour fcc or hcp hollow sites in the (111) surface (Fig. 6e). Their distance amounts to $4.3 \AA$, close enough to enable significant overlap of the Au orbitals but sufficiently far to coordinate the two S atoms of the thioether simultaneously. The BPPS monomer is therefore stabilized by two $\mathrm{Au}-\mathrm{S}$ and one $\mathrm{Au}-\mathrm{Au}$ bond. We note that incorporating metal dimers into a molecular assembly is a common motive as it increases the structural flexibility of the metal-organic frame. ${ }^{17,27}$

Also the S-atom in the centre of the BPPS monomer can be identified in the conductance maps. At $-1.7 \mathrm{~V}$, a single maximum becomes visible opposite to the Au pair. We assign this protrusion to the lone-pair state of the second S atom that is expected to be below the Fermi level. The fact that the same state is not detected for the other two sulphurs indicates their different binding situation due to the direct contact with the $\mathrm{Au}$ atoms. Once all $\mathrm{S}$ centres plus the coordinated $\mathrm{Au}$ atoms are identified, a binding geometry can be proposed for the BPPS monomer (Fig. 6e). The molecule winds around the Au pair, forming covalent $\mathrm{Au}-\mathrm{S}$ bonds with the first and third $\mathrm{S}$ centre. The second $\mathrm{S}$ does not coordinate an adatom, but either binds to the metal support or dangles in the vacuum. We suspect that also the benzene rings contribute to the image contrast, producing the halo around the Au dimer, but have no information on their precise geometry. Whereas a parallel orientation with respect to $\mathrm{Au}(111)$ would maximize the dispersive and $\pi$-mediated forces, a tilted geometry would soften the geometric constraints put forth by the $\mathrm{Au}-\mathrm{S}$ bonds.

A similar analysis has been carried out for other BPB and BPPS complexes on the $\mathrm{Au}(111)$ surface and is summarized in Fig. 7. The dim features of Fig. 3a are all assigned to bare molecules that were unable to catch a lattice gas atom. We believe that the contrast in this case is dominated by flat-lying benzene rings, following the interpretations of the Yates and Weiss groups. ${ }^{30,31}$ In those studies, flat-lying benzene rings were imaged as $1.2 \AA$ high protrusions, while the $\mathrm{S}$-atoms were found to elevate by less than $0.9 \AA$ above the surface. Strongly inclined phenyl rings, on the other hand, are hardly contributing

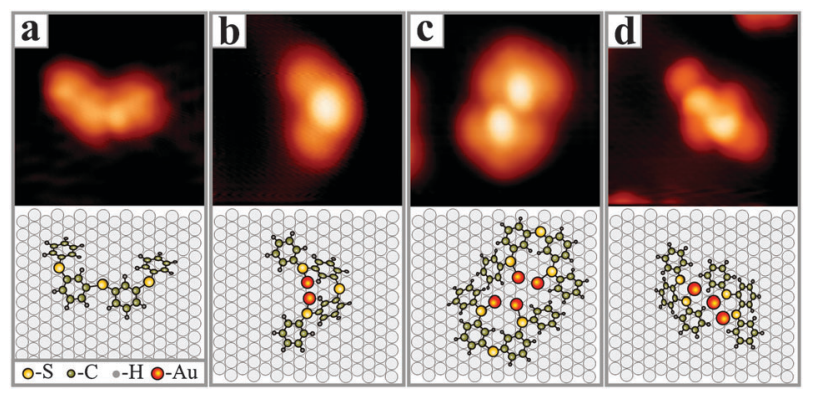

Fig. 7 Topographic images and corresponding structure models of different thioether complexes on $\mathrm{Au}(111)\left(4 \times 4 \mathrm{~nm}^{2}, U_{\mathrm{s}}=-0.5 \mathrm{~V}\right)$. (a) Bare BPPS, (b) elongated BPPS monomer, (c) oval BPPS dimer and (d) BPB dimer. to the images, because their $\pi$-electronic system lies perpendicular to the surface and overlaps only little with the substrate electronic states. ${ }^{17}$ On this basis, a tentative binding geometry has been developed for the bent BPPS molecule (Fig. 7a). The contrast is governed by the two central phenylthio units, while the outer rings are hardly visible due to their potential tilt. In a similar way, the appearance of bare BPB and straight BPPS conformers might be rationalized as well. We note that these models are necessarily tentative because no information on the orientation of the phenyl rings is available at this point.

However, most of the ligand complexes are characterized by one or two central protrusions, being ascribed to coordinated $\mathrm{Au}$ atoms. In addition to the oval BPPS monomer that has been discussed above, we present a possible binding geometry for the elongated BPPS monomer in Fig. 7b. The protrusion in the centre is again assigned to an $\mathrm{Au}$ dimer bound to the first and third S-atom, while the faint maxima at both ends mark the flat-lying peripheral phenyl rings of the molecule. The fact that the central phenylthio unit does not leave a clear topographic fingerprint is explained with its negligible overlap with the gold electronic states, for instance due to a tilted or elevated binding position. Finally, the ad-structures presented in Fig. 3c have been related to dimer complexes that contain two molecules inter-connected by several Au atoms. A plausible binding geometry for a BPPS dimer has been obtained by approaching two monomer complexes until an additional $\mathrm{Au}-\mathrm{Au}$ bond forms across both units (Fig. 7c). While the individual BPPS- $\mathrm{Au}_{2}$ assemblies preserve their original configuration, the additional $\mathrm{Au}-\mathrm{Au}$ bond stabilizes the dimer with respect to the isolated monomers. A similar picture can be obtained for the BPB dimer, except that three instead of four Au adatoms are required to link both molecules (Fig. 7d). Experimentally, this difference becomes evident from the brighter appearance of the lower right compared to the upper left protrusion of the complex. From a structural point of view, it reflects the reduced complexation potential of BPB with two S-centres compared to BPPS that contains three sulphurs. In general, the abundance of dimers with respect to monomer species provides evidence for the exothermic nature of such dimerization processes, but also suggests a certain mobility of the monomer units at room temperature.

At higher exposure, elongated chains develop on the $\mathrm{Au}(111)$, being composed of the dimer units discussed before. The chain formation becomes possible only if the thioether molecules fully exploit their complexation potential, that means, if each sulphur in the molecule coordinates an $\mathrm{Au}$ atom. Those extra atoms permit the interaction between neighbouring complexes, either via $\mathrm{Au}-\mathrm{Au}$ bonds as shown for the dimer or via hydrogen bonds between $\mathrm{Au}$ and $\mathrm{H}$ atoms in adjacent molecules. Given the structural complexity and the lack of atomic-scale information, we abstain from developing binding models for the BPB and BPPS chains. We note however that only the elongated BPPS dimer is able to agglomerate into a chain, while the oval one exclusively appears at the chain ends or as isolated species. This observation might be explained by the good shielding of the Au dimer by the enclosing molecule in the oval BPPS configuration.

In summary, the adsorption of both thioether molecules on $\mathrm{Au}(111)$ is mediated by mobile adatoms provided by the roomtemperature lattice-gas. The metal coordination is driven by the 
formation of strong $\mathrm{Au}-\mathrm{S}$ bonds. In most cases, $\mathrm{Au}$ dimers link the individual molecules, as this increases the structural flexibility of the metal-organic complexes and reduces the effect of steric repulsion. We note that an inter-molecular coupling via metal dimers has been observed for other thioethers, ${ }^{17}$ and is a common motive in metal-organic networks. ${ }^{27}$

A rather different binding behaviour is revealed for BPB and BPPS on $\mathrm{NiAl}(110)$, where typical adsorbates only protrude by $1.5 \AA$ from the metal surface. Whereas this value is compatible with the imaging contrast induced by flat-lying benzene rings and $\mathrm{S}$-atoms, ${ }^{30,31}$ it is clearly too small to represent metal atoms in a molecular complex. Note that already isolated adatoms appear as $2 \AA$ tall protrusions on the NiAl surface. ${ }^{32}$ The absence of comparable maxima in our study makes us believe that no metal complexation takes place and only the bare BPB and BPPS molecules are detected on the surface. To support this conclusion, we note that thioether molecules of reduced height have also been observed on a $\mathrm{FeO}(111)$ surface, where mobile metal atoms are clearly absent. ${ }^{17}$ Two reasons are conceivable to explain the different adsorption properties with respect to $\mathrm{Au}(111)$, namely the absence of a room-temperature lattice gas and the inability of the thioether species to stabilize $\mathrm{Ni}$ or $\mathrm{Al}$ atoms. We discard the latter option, because both $\mathrm{Ni}$ and $\mathrm{Al}$ are known to form stable, high-melting compounds with sulphur, such as NiS and $\mathrm{Al}_{2} \mathrm{~S}_{3}$. Complexation reactions are thus expected to take place if only the metal adatoms would be available on the surface. We thus suspect that no or few lattice-gas atoms are present on $\mathrm{NiAl}(110)$ at room temperature, a phenomenon that might be rationalized with the following arguments. The mixed $\mathrm{NiAl}(110)$ termination with its specific binding sites for $\mathrm{Ni}$ and $\mathrm{Al}$ renders diffusion and reintegration of adatoms into the step edges difficult. Also, the diffusion barriers are higher than on the closed-packed $\mathrm{Au}(111)$ because of an extra surface corrugation generated by the outward relaxation of $\mathrm{Al}$ with respect to $\mathrm{Ni}^{24,33}$ In the bulk limit, the $\mathrm{Ni}-\mathrm{Al}$ bond is stronger than the $\mathrm{Au}-\mathrm{Au}$ bond, as deduced from the higher melting temperature of the alloy crystal. And finally, the NiAl step edges might be stabilized by small quantities of water and oxygen from the rest gas, an effect that can be excluded for the inert gold surface. ${ }^{34}$

The ad-features found on the $\mathrm{NiAl}(110)$ are therefore taken as topographic fingerprints of pristine BPB and BPPS molecules. In both cases, the flat-lying benzene rings and the S-centres may contribute equally to the contrast, rendering an unambiguous identification of the binding geometry difficult. In $\mathrm{BPB}$, the $\mathrm{S}-\mathrm{S}$ distance closely matches the $\mathrm{NiAl}[110]$ unit-cell vector of $4.1 \AA$. It is therefore reasonable to assume that the two sulphurs attach to equivalent positions in adjacent $\mathrm{Ni}$ or $\mathrm{Al}$ rows with the formation of Ni-S bonds being more likely. Such a binding scenario would explain the [110]-orientation and the bar-shape of the molecules in the topographic images (Fig. 8a). While the outer protrusions are assigned to the two peripheral phenyl rings, the maximum in the middle might reflect the phenylthio unit in the centre of the BPB. Note that only two S-atoms are detected for specific tip configurations, and the molecules appear as simple double protrusions in this case (Fig. 5c, lower left panel).

A similar binding geometry is suggested for the BPPS, except that the molecule appears longer and wider than its

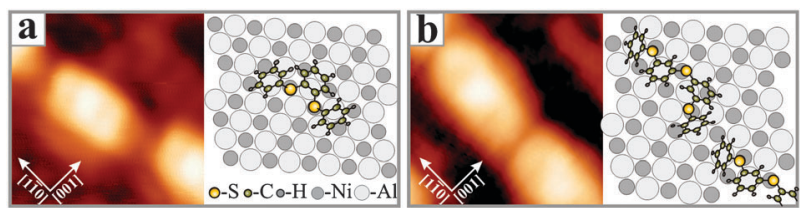

Fig. 8 Topographic images and corresponding structure models of (a) BPB and (b) BPPS molecules on $\operatorname{NiAl}(110)\left(2 \times 2 \mathrm{~nm}^{2},-0.5 \mathrm{~V}\right)$.

BPB counterpart due to the presence of three S-centres. The enlarged S-S distance in BPPS favours an alignment of the phenylthio units along the diagonal of the NiAl unit cell and not along the [110] direction (Fig. 8b). The three maxima detected along the molecular axis are again ascribed to the two outer phenyl rings and the central diphenylthio unit that appears brighter. We note that also a shorter, two-centre species is found on the NiAl surface that might be assigned to a BPPS whose outer phenyls have completely rotated out of the surface plane and are thus invisible in the STM. Apparently, this configuration is adopted in the molecular superstructure that forms by stacking [110]-oriented molecules along the [001] direction (Fig. 5d, right panels). The intermolecular interaction within the chains is likely mediated by hydrogen bonds formed between an S-atom of one and $\mathrm{H}$-atoms of the adjacent molecule. The commensurability of the superstructures with respect to the $\mathrm{NiAl}$ lattice indicates that molecule-support and not intermolecular coupling governs the spatial arrangement of the thioether species in this particular case.

\section{Summary}

We have demonstrated that the adsorption behaviour of sulphur-containing benzene-derivatives does not only depend on the arrangement of aryl groups and bridging S-atoms, but much more on the properties of the substrate. On $\mathrm{Au}(111)$, well-defined metal-organic complexes are formed by coordinating mobile $\mathrm{Au}$ atoms from the lattice-gas to the S-centres of the molecule. In contrast, only the pristine molecules are detected on $\mathrm{NiAl}(110)$, where only a few adatoms are available at room temperature. These adsorption peculiarities visualize how important certain surface properties, i.e. the attachment/detachment equilibrium of atoms from surface step edges, are for the interaction with organic molecules. The exact molecular constitution, on the other hand, plays only a minor role, as long as similar building blocks are present in the different species. This becomes evident from the comparable appearance of BPB and BPPS molecules in STM images taken on both metal substrates.

Our study confirms the large affinity of thioether molecules to bind single metal adatoms. A high structural flexibility of the molecular frame is however required to reach the maximum coordination number. In our study, BPB and BPPS monomers were found to bind two Au atoms each, a number that increases to four and probably six in the respective dimer and chain structures. Given their ability in stabilizing metal atoms, both thioether molecules are well suited to control ripening and sintering processes on catalytically relevant surfaces. In such applications, the molecules may disrupt the material transport between the chemically active metal centres, preserving their initial size and shape. To explore molecule-driven stabilization 
effects in a catalytically-relevant environment, our adsorption experiments need to be repeated on an oxide surface covered with $\mathrm{Au}$ deposits. The Au(111) lattice gas is replaced in this case by atom diffusion between the different particles, while the nature of coordination reactions is believed to be unchanged.

\section{Acknowledgements}

This work has been supported by the DFG through the 'Cluster of Excellence UNICAT'.

\section{Notes and references}

1 G. E. Poirier and E. D. Pylant, Science, 1996, 272, 1145.

2 S. M. Driver and D. P. Woodruff, Surf. Sci., 2000, 457, 11-23; D. P. Woodruff, Phys. Chem. Chem. Phys., 2008, 10, 7211.

3 E. Pensa, P. Carro, A. A. Rubert, G. Benítez, C. Vericat and R. C. Salvarezza, Langmuir, 2010, 26, 17068.

4 P. Maksymovych, D. C. Sorescu and J. T. Yates, Phys. Rev. Lett., 2006, 97, 146103.

5 J. A. Boscoboinik, R. R. Kohlmeyer, J. Chen and W. T. Tysoe, Langmuir, 2011, 27, 9337.

6 M. Yu, N. Bovet, C. J. Satterley, S. Bengió, K. R. J. Lovelock, P. K. Milligan, R. G. Jones, D. P. Woodruff and V. Dhanak, Phys. Rev. Lett., 2006, 97, 166102.

7 P. Maksymovych, O. Voznyy, D. B. Dougherty, D. C. Sorescu and J. T. Yates, Prog. Surf. Sci., 2010, 85, 206.

8 A. Chaudhuri, D. C. Jackson, T. J. Lerotholi, R G. Jones, T.-L. Lee, B. Detlefs and D. P. Woodruff, Phys. Chem. Chem. Phys., 2010, 12, 3229.

9 Y. Wang, N. S. Hush and J. R. Reimers, J. Am. Chem. Soc., 2007, 129, 14533.

10 F. Li, L. Tang, W. Zhou and Q. Guo, J. Am. Chem. Soc., 2010, 132, 13059.

11 J. V. Barth, G. Costantini and K. Kern, Nature, 2005, 437, 671; A. Dmitriev, H. Spillmann, N. Lin, J. V. Barth and K. Kern, Angew. Chem., Int. Ed., 2003, 42, 2670.

12 S. L. James, Chem. Soc. Rev., 2003, 32, 276.

13 D. Zacher, O. Shekhah, C. Wöll and R. A. Fischer, Chem. Soc. Rev., 2009, 38, 1418.
14 H. Walch, J. Dienstmaier, G. Eder, R. Gutzler, S. Schlogl, T. Sirtl, K. Das, M. Schmittel and M. Lackinger, J. Am. Chem. Soc., 2011, 133, 7909 .

15 R. D. Vengrenovitch, Acta Metall., 1982, 30, 1079; A. Baldan, J. Mater. Sci., 2002, 37, 2171.

16 G. Ertl, H. Knözinger, F. Schueth and J. Weitkamp, Handbook of heterogeneous catalysis, Wiley-VCH, Weinheim, 2nd edn, 2008.

17 B. Yang, X. Lin, Y. Pan, N. Nilius, H.-J. Freund, C. Hulot, A. Giraud, S. Blechert, S. Tosoni and J. Sauer, J. Am. Chem. Soc., 2011, DOI: 10.1021/ja300304s.

18 R. C. Jaklevic and L. Ehe, Phys. Rev. Lett., 1988, 60, 120; M. Baba, A. Natori and H. Yasunaga, Surf. Sci., 1990, 239, 363.

19 K. Morgenstern, G. Rosenfeld and G. Comsa, Phys. Rev. Lett., 1996, 76, 2113

20 W. Reifschneider, US Pat., 3102916 19630903, 1963; R. Adams, W. Reifschneider and A. Ferreti, Org. Synth., 1973, 5, 107.

21 T. Yusaku and I. Yukio, JP Pat., 2001302615 A 20011031, 2001; R. Varala, E. Ramu, M. Alam Mujahid and S. R. Adapa, Chem. Lett., 2004, 33, 1614.

22 C. G. Bates, R. K. Gujadhur and D. Venkataraman, Org. Lett., 2002, 4, 2803.

23 N. Hiroshi, N. Yoshinobu and K. Isao, JP Pat., 10017543 A 19980120, 1998

24 H. L. Davis and J. R. Nooman, Phys. Rev. Lett., 1985, 54, 566.

25 Remember that the STM contrast does not reflect the true sample topography but only the state density that is available for tunneling. Protruding features with negligible electronic overlap to states in the support are hence undetectable with STM.

26 We note that a removal of the $\mathrm{Au}(111)$ herringbone reconstruction may also provide single $\mathrm{Au}$ atoms for coordination. We disfavor this scenario, as the typical signature of the reconstruction remains visible in the STM after molecular dosage.

27 J. V. Barth, Annu. Rev. Phys. Chem., 2007, 58, 375.

28 S. Tosoni, A. D. Boese and J. Sauer, J. Phys. Chem. C, 2011, 115, 24871.

29 N. Nilius, T. M. Wallis, M. Persson and W. Ho, Phys. Rev. Lett., 2003, 90, 196103.

30 P. Han, B. A. Mantooth, E. C. H. Sykes, Z. J. Donhauser and P. S. Weiss, J. Am. Chem. Soc., 2004, 126, 10787.

31 P. Maksymovych and J. T. Yates, J. Am. Chem. Soc., 2008, 130, 7518.

32 T. M. Wallis, N. Nilius and W. Ho, J. Chem. Phys., 2003, 119, 2296.

33 Y. Mishin, A. Y. Lozovoi and A. Alavi, Phys. Rev. B: Condens. Matter Mater. Phys., 2003, 67, 014201.

34 S. Ulrich, N. Nilius and H. J. Freund, Surf. Sci., 2007, 601, 4603. 